\title{
Impact of the COVID-19 pandemic on the production and sales of cars in the world
}

\author{
Shevchenko I. Y. ${ }^{1}$, Dmytriiev I. A. ${ }^{1}$, Dmytriieva O. I. ${ }^{1}$ \\ ${ }^{1}$ Kharkiv National Automobile and Highway University, Ukraine
}

\begin{abstract}
Annotation. Problem. The global automotive industry has already had an experience of recovery from the global financial crisis of 2008, but the pandemic crisis of 2020 is quite different in nature and pattern of progress: in recent history it has had no analogues and it will be premature to state its completion. Therefore, it is important to determine the impact of the pandemic on the production and sale of cars in order to overcome the negative consequences. To address this issue, the article identifies the sensitivity of this subsector of mechanical engineering to destructive changes in the environment; an analysis of changes in the volume of production and sales of cars by countries of the world over the past period has been made. Goal. The aim of the work is to determine the destructive consequences and trends of the COVID-19 pandemic impact on the global automotive industry, namely the production and sale of cars. Methodology. To determine the impact of the COVID-19 pandemic, a vertical and horizontal analysis of car production and sales in the world has been conducted. Results. The results of the analysis allowed the authors to group the countries of the world by the destructive effects of the pandemic crisis of 2020 for the automotive industry. Originality. The carried out classification of countries by the destructive effects of the COVID-19 pandemic provided an opportunity to gain insight into its impact on the automotive industry, in particular on the production and sale of cars. Practical value. The obtained results can be recommended to identify further ways to overcome the negative effects of the COVID-19 pandemic in the automotive industry.
\end{abstract}

Key words: automotive industry, socio-economic development, car production, car sales, COVID-19 pandemic.

\section{Introduction}

A strategically important part of the national economy, industry and machine-building complex is the automotive industry - a powerful generator of an added value and a flagship of the innovation activity. It is believed that the progress of the national economy and its place on the world economic arena is largely determined by the presence in its structure of space industry, aircraft and automotive industry, because these industries provide a powerful impetus to the development of science and technology, and building a network of enterprises in related industries. For example, the creation of 1 job in the automotive industry leads to the creation of at least 6 jobs in related industries. Such synergy promotes the development of specialization and cooperation, the implementation of cluster initiatives at the mesolevel on the basis of public-private partnership.

But not only car production characterizes the socio-economic development of the country: an important indicator is the sales of new cars, which indicate the material security of the population in terms of its paying capacity in the long term period.

Since December 2020, all sectors of the world's national economies have faced a huge challenge and come under the influence of the World Coronavirus Epidemic, called COVID-19. The pandemic has already affected the paying capacity of the population and, consequently, its buying capacity.

The global automotive industry has already had an experience of recovery from the global financial crisis of 2008, but the pandemic crisis of 2020 is quite different in nature and pattern of progress: in recent history it has had no analogues and it will be premature to state its completion.

Therefore, it is important to determine the impact of the pandemic on the production and sale of cars in order to overcome the negative consequences. 


\section{Analysis of publications}

The impact of the COVID-19 pandemic is being hotly debated not only in the scientific community but in society as a whole. The tough economic conditions of the World Epidemic have prompted the scientists to study trends in its impact, implications for national economies, and ways out of the crisis.

In the study [1] the author has analyzed the state and trends of the impact of COVID-19 on the economic situation in the world, in particular, the definition of socio-economic losses. The scientist has conducted a study of the results of Rosamund Hutt's analysis for the WEF, where he has found a decline in all indicators of economic growth in the world. The study shows the expected reduction in employment in terms of sectors of the national economy, due to the introduction of quarantine in Ukraine. The scenarios of the global crisis proposed by the world experts and politicians deserve attention and further research.

The author [2] has considered the main trends in the global automotive industry on the example of three main markets for this sector, namely the European region, the United States and China. The study has identified the characteristic aspects of the dynamics of automotive production during the pandemic of coronavirus pneumonia COVID-19. The author has analyzed the indicators of car sales by geographical area in the frame of epidemic spread and relevant regulatory measures.

In Ukraine, the research [3] was presented in 2020. It was prepared by the NGO "Center for Applied Research" with the support of the Konrad Adenauer Foundation Representation in Ukraine. The authors of the study "The impact of COVID-19 and quarantine restrictions on the economy of Ukraine" have analyzed the internal and external factors of the crisis in the economy. The study has analyzed the impact of quarantine measures on certain sectors of Ukraine's economy, including transport. But this study did not take into account the analysis of the demand for the cars.

Foreign scientists also pay enough attention to the impact of the World Pandemic on the indicators of economic growth of world's national economies [4-12].

In the studies [6-8] the authors have identified the impact of the pandemic crisis on all sectors of the national economy, and also they have proposed the measures to stabilize the situation.

The authors [5] on the example of Saudi Arabia have conducted a study on the impact of COVID-19 on entrepreneurship and consumer behavior.

Much attention has also been paid to the study of the impact of the pandemic on the automotive industry by foreign scientists. So, for example, the works [4.9-13] are aimed at highlighting the current consequences of the pandemic and measures to overcome them in the automotive industry of different countries.

\section{Purpose and Tasks}

The aim of the study is to determine the destructive consequences and trends of the COVID-19 pandemic impact on the global automotive industry, namely the production and sale of cars.

In accordance with the desired goal it is necessary to determine the sensitivity of this subbranch of machine-building to destructive changes in the external environment; to conduct a vertical and horizontal analysis of changes in the volume of production and sales of cars in the world in recent years.

Despite the undeniable advantages of the development of the automotive industry in the country, it is fair to note the increased sensitivity of this subsector of mechanical engineering to destructive changes in the external environment. The last twenty years have been marked by two global crises - the financial crisis of 2008 and the pandemic crisis of 2020. The global automotive industry has naturally responded to the crisis by reducing the scale of activity (Fig. 1, made by the authors according to [14-15]).

According to preliminary estimates, the pandemic crisis of 2020 will have more destructive consequences for the global automotive industry than the global financial crisis of 2008: for example, in the crisis year of 2008 the decline in car production was $3.46 \%$ (including a reduction in passenger car production $-0,68 \%$, a reduction in the production of commercial vehicles $10.85 \%$ ), while in the pandemic year of 2020 , the volume of car production decreased by $15.43 \%$ (including the volume of passenger car production - by $16.85 \%$, the volume of commercial vehicles production - by $11.57 \%$ ). 
120000000

pandemic crisis

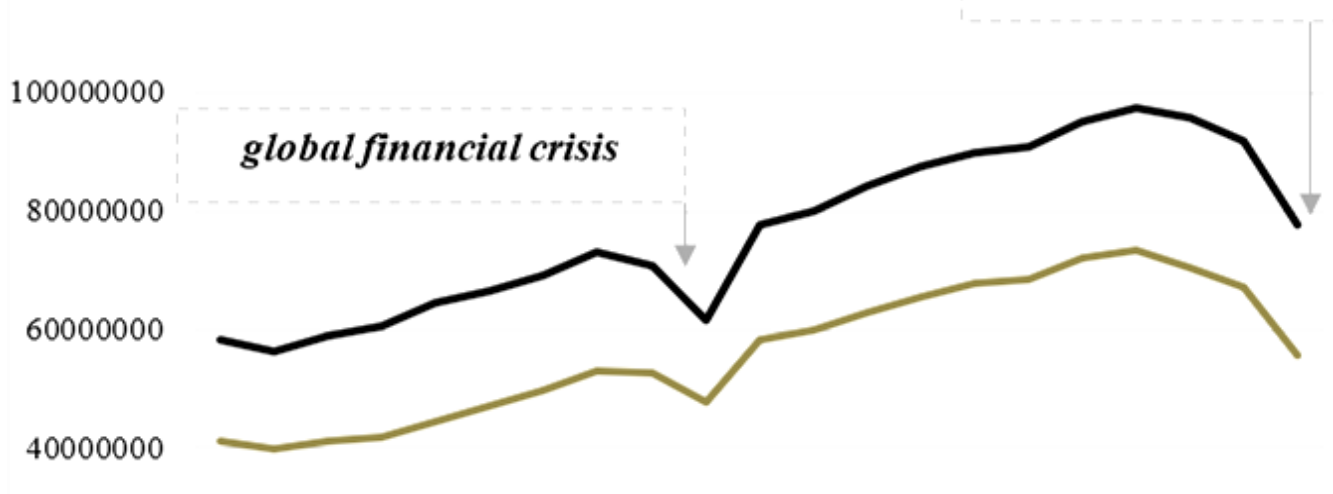

20000000

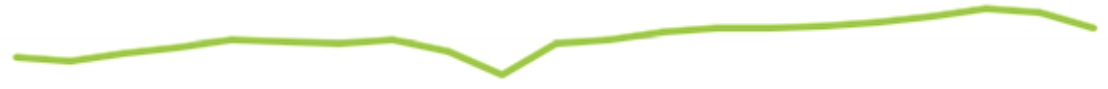

0

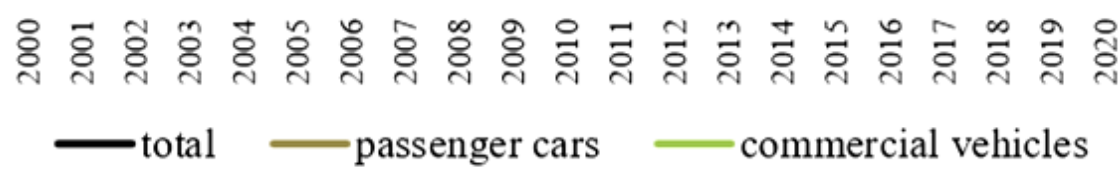

Fig. 1. Production of cars (including cars and commercial vehicles) in the world in 2000-2020

For the analysis we will choose the period of 2019-2020 and perform a vertical and horizontal analysis of changes in production and sales of cars around the world (Table 1-2, [calculated by the authors according to: [14]).

The results of the vertical analysis of the volumes of car production in the world give us opportunity to state that the flagships of the global automotive industry are China, USA, Japan, Germany, India, Mexico, South Korea, Brazil, Spain, France. These countries have produced $77.75 \%$ of all cars (including $77.22 \%$ of cars and $79.21 \%$ of commercial vehicles) in 2019 and $79.27 \%$ of all cars (including $78.38 \%$ of cars and $81.55 \%$ of commercial vehicles) in 2020. The results of the vertical analysis of car sales in the world have shown the leadership of such countries as China, USA, Japan, Germany, India, Brazil, France, which together account for more than $65 \%$ of all sold cars.

Based on the results of the horizontal analysis of car production and sales in the world, we will classify the countries of the world by the destructive effects of the pandemic crisis of 2020 for the automotive industry by criterion of reducing car production and sales (Table 3), where the reduction: by a percentage not exceeding $5 \%$, can be identified as minor destructive effects; by a percentage in the range of 5-15\% - as quite significant destructive consequences; by a percentage in the range of 15-25\% - as significant destructive consequences; by a percentage exceeding $25 \%-$ as very significant destructive consequences.

According to table 3 we can see that the most destructive effects of the pandemic crisis of 2020 have affected the automotive industries of Canada, Thailand, France, South Africa, Indonesia, Austria, Brazil, Poland, the United Kingdom, Morocco, Serbia, Slovenia, Mexico, Portugal. India, and Malaysia. In the context of the analysis of car sales, the most striking signs of destruction are observed in countries such as Croatia, Spain, Portugal, Mexico, Chile, Peru, Indonesia, Philippines, South Africa, Bulgaria, Greece, Italy, United Kingdom, Brazil, Colombia, Ecuador, Pakistan, UAE, Slovakia, France, Canada, USA, Kuwait, Thailand, Romania, Slovenia, Sweden, and India.

It is fair to note the fact that in the pandemic year of 2020, still, there were isolated cases of growth in production and sales of cars in some countries. In 2020, car production increased in Egypt (by $28.4 \%$ ), Iran (by $7.3 \%$ ) and Uzbekistan (by $3.3 \%$ ), including: car production increased in Egypt by $28.4 \%$, Iran - by $7.3 \%$, Uzbekistan - by $3.3 \%$. Production of commercial vehicles increased in Germany (by $100 \%$ ), China (by $19.97 \%$ ), Taiwan (by $4.68 \%$ ) and Iran (by $7.3 \%)$. 
Table 1. Vertical and horizontal analysis of car production (including cars and commercial vehicles) in the world in 2019-2020

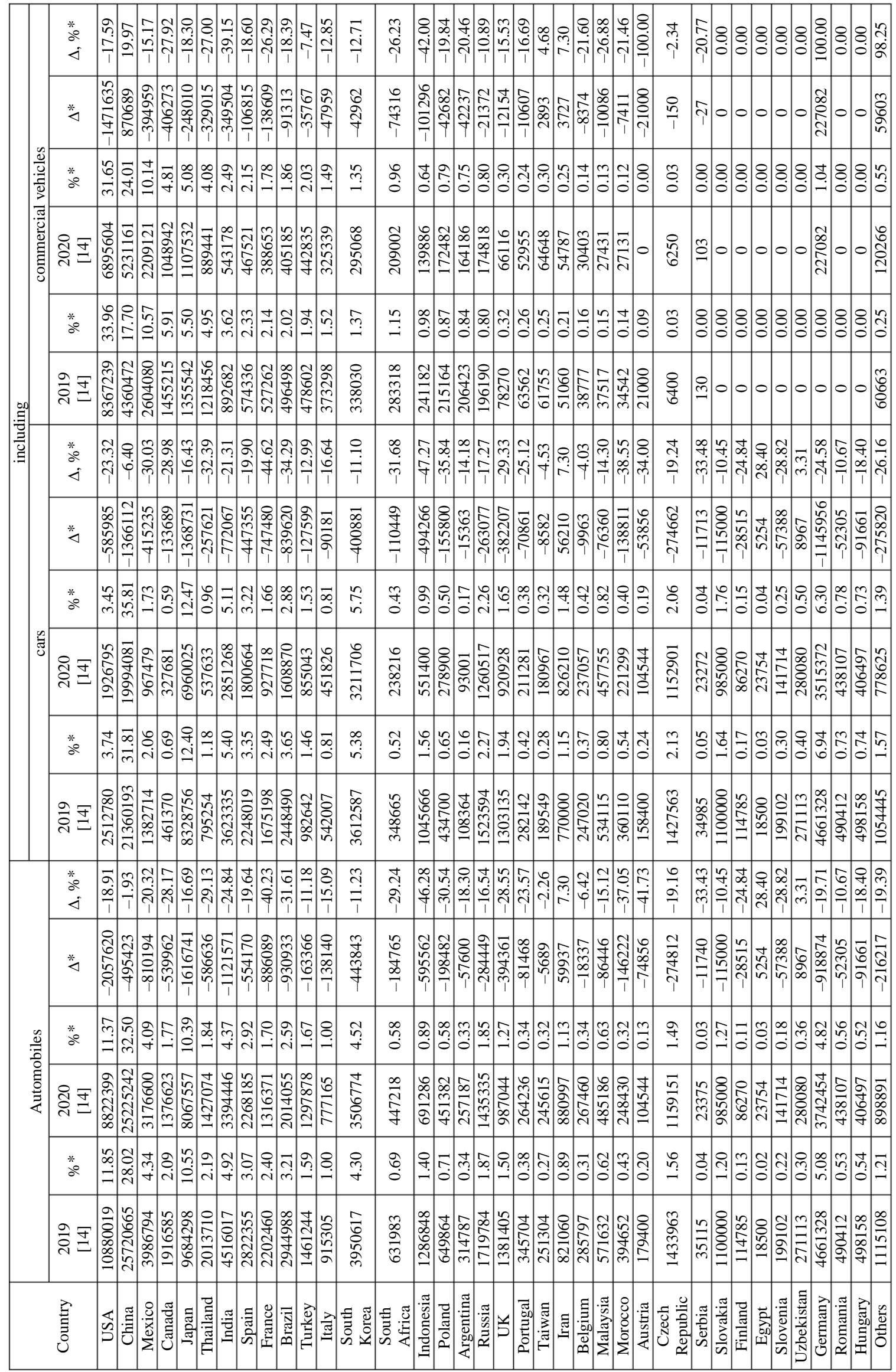


Table 2. Vertical and horizontal analysis of car sales (including cars and commercial vehicles) in the world in 2019-2020

\begin{tabular}{|c|c|c|c|c|c|c|c|c|c|c|c|c|c|c|c|c|c|c|c|c|c|c|c|c|c|c|c|c|c|c|c|c|c|c|c|c|c|c|c|c|c|}
\hline & $a_{j}^{*}$ & 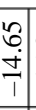 & 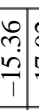 & & & & $=$ & tis & & \begin{tabular}{l|l}
8 \\
\hdashline \\
1
\end{tabular} & $\begin{array}{ll}7 \\
7 \\
1\end{array}$ & & $\frac{7}{1}$ & $\frac{\infty}{2}$ & \begin{tabular}{l}
$\infty$ \\
\multirow{1}{*}{} \\
$\infty$ \\
$\uparrow$
\end{tabular} & & $\begin{array}{l}0 \\
\delta \\
0 \\
0 \\
1 \\
\end{array}$ & 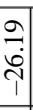 & $\begin{array}{c}+ \\
\infty \\
\infty \\
\infty \\
1 \\
\end{array}$ & $\begin{array}{l}0 \\
\\
\end{array}$ & 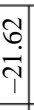 & & & & & & & & & & & & & & & & & 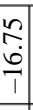 & & & \\
\hline & $\stackrel{*}{4}$ & $y$ & J & & & & $\frac{1}{1}$ & h. & & & 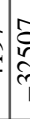 & & & $\infty$ & 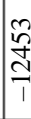 & & 命 & 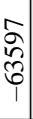 & 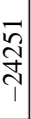 & 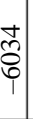 & 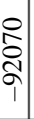 & & & & & & & & & & a & $\underline{\varepsilon}$ & & & & & & ڤัे & & & \\
\hline $\begin{array}{l}\frac{0}{0} \\
\frac{0}{2} \\
\frac{0}{0}\end{array}$ & & $\overrightarrow{0}$ & ?.: & & & & & & & & & & & & & & & & & $\begin{array}{l}0 \\
0 \\
0\end{array}$ & & & & & & $\infty$ & & & & & in & & & & & & & $\begin{array}{l}+ \\
\infty \\
- \\
-\end{array}$ & & & \\
\hline & $\sqrt{5}$. & 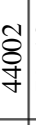 & $\frac{\infty}{\square}$ & & & & 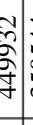 & & & & & & & & & & & $\mid$ & $\frac{a}{\infty}$ & 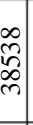 & હે & & & & . & f & & & & & $\approx$ & & & & & $\ddot{n}$ & & 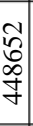 & $\begin{array}{c}0 \\
\infty \\
\infty \\
0 \\
0\end{array}$ & & \\
\hline & ${ }^{*}$ & $\begin{array}{l}\frac{9}{0} \\
0\end{array}$ & ?ి: & $\begin{array}{l} \pm \\
\\
0\end{array}$ & \pm & & & 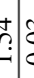 & & 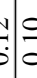 & & 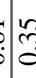 & $\frac{1}{0}$ & $\overbrace{\infty}^{\infty}$ & & $\stackrel{0}{0}$ & ô. & $\bar{g}$ & $\begin{array}{l}\hat{n} \\
0\end{array}$ & $\begin{array}{l}5 \\
0 \\
0\end{array}$ & : & $\stackrel{2}{0}$ & & : & & & & & & & 0 & $\stackrel{m}{m}$ & $\stackrel{2}{0}$ & & & ஸे & & ฉ & $\stackrel{m}{0}$ & & \\
\hline : & 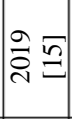 & $\begin{array}{l}n \\
n \\
n \\
n\end{array}$ & & & & & 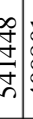 & 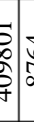 & & & & & 齐 & ह & & & 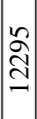 & 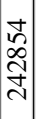 & 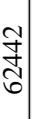 & 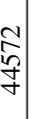 & 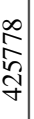 & 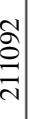 & & $\frac{6}{ \pm}$ & 合 & h) & & $\begin{array}{c}6 \\
0 \\
a \\
c\end{array}$ & & & 竎 & $\infty$ & $\frac{尺}{\stackrel{9}{J}}$ & & & $\begin{array}{l}\bar{n} \\
\text { ñ } \\
\text { n. }\end{array}$ & & 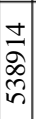 & 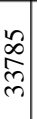 & & $\infty$ \\
\hline & $\begin{array}{l}* \\
\partial^{\circ} \\
\dot{\gamma}\end{array}$ & 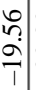 & 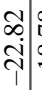 & 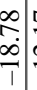 & $\begin{array}{l}\vec{y} \\
\mathrm{i} \\
\end{array}$ & $\begin{array}{l}0 \\
0 \\
\vdots \\
1 \\
1\end{array}$ & 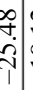 & 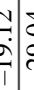 & 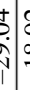 & 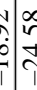 & & $\frac{1}{\frac{1}{2}}$ & $\frac{\pi}{T}$ & ๙ิ & $\begin{array}{l}\delta \\
\infty \\
\infty \\
1\end{array}$ & $\frac{2}{i}$ & $\begin{array}{l}\hat{\infty} \\
\stackrel{+}{\uparrow} \\
\uparrow\end{array}$ & 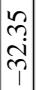 & \begin{tabular}{l}
$\infty$ \\
$\vdots$ \\
\\
\hdashline
\end{tabular} & 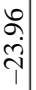 & 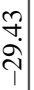 & & & & 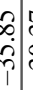 & 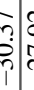 & & & & & $\begin{array}{l}8 \\
7 \\
21\end{array}$ & $\stackrel{\text { I }}{=}$ & $\begin{array}{l}\overline{\text { }} \\
\text { ָे }\end{array}$ & & & $\begin{array}{l}\infty \\
\stackrel{\infty}{\sim} \\
i \\
T\end{array}$ & & $\begin{array}{l}\stackrel{?}{0} \\
\dot{0} \\
\stackrel{1}{1}\end{array}$ & $\begin{array}{l}\hat{n} \\
\stackrel{n}{+} \\
\end{array}$ & & \\
\hline & $\|^{*}$ & 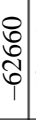 & 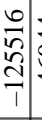 & & $\begin{array}{c}\vec{T} \\
\stackrel{\sim}{\top} \\
\top\end{array}$ & $\frac{0}{\infty}$ & 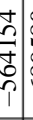 & 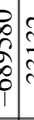 & $\begin{array}{l}v \\
\hat{n} \\
\hat{n} \\
\hat{n}\end{array}$ & & & $\left\{\begin{array}{l}0 \\
\infty \\
\infty\end{array}\right.$ & \begin{tabular}{l}
9 \\
$\vdots$ \\
\hdashline \\
\hdashline
\end{tabular} & 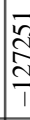 & & $\begin{array}{l}= \\
\bar{\sim} \\
\text { } \\
1\end{array}$ & 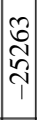 & \begin{tabular}{|l|}
0 \\
0 \\
0 \\
0 \\
$⿱ 亠$ \\
1 \\
\end{tabular} & 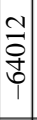 & 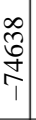 & 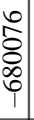 & 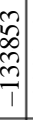 & & & 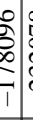 & 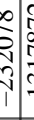 & 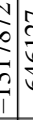 & 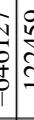 & 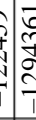 & & 1) & $\begin{array}{l} \pm \\
\bar{\sigma} \\
\end{array}$ & 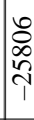 & స్ & 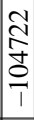 & & & 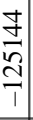 & $\begin{array}{l}0 \\
\frac{a}{0} \\
0 \\
0\end{array}$ & $\begin{array}{l}\hat{\delta} \\
\stackrel{\infty}{6} \\
\frac{6}{1}\end{array}$ & $\begin{array}{l}\infty \\
\infty \\
\end{array}$ \\
\hline & 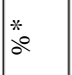 & $\stackrel{\infty}{\stackrel{\infty}{0}}$ & $\stackrel{?}{\circ}$ & $\stackrel{\infty}{3}$ & $\widehat{c}$ & & : & t. & 9 & $\vec{t}$ & & 0 & $\hat{n}$ & 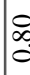 & $\stackrel{\overbrace{}}{\curvearrowright}$ & 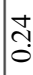 & $\frac{ \pm}{0}$ & -1 & 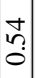 & t. & 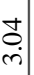 & 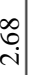 & \pm & $\frac{6}{0}$ & ?. & b. & 6 & & & & $\frac{2}{2}$ & $\bar{r}$ & \pm & & & $\stackrel{N}{0}$ & $\begin{array}{c}\sigma \\
\dot{\delta} \\
\dot{m}\end{array} \mid$ & 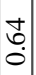 & 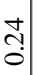 & $\begin{array}{l}0 \\
0 \\
0\end{array}$ & in \\
\hline & 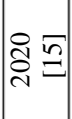 & $\begin{array}{c}\overrightarrow{\mathbf{v}} \\
\stackrel{\widehat{n}}{\mathrm{a}} \\
\end{array}$ & & & 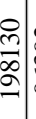 & مิ & 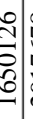 & 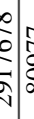 & $=$ & $\vec{c}$ & 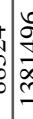 & 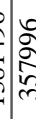 & I & 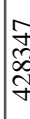 & 孚 & 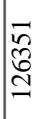 & 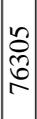 & 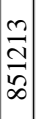 & 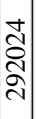 & 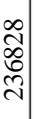 & 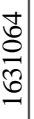 & 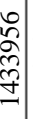 & & & 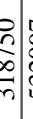 & 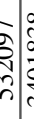 & 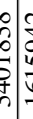 & 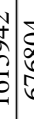 & & & $\begin{array}{l}2 \\
\vdots \\
\infty \\
\infty \\
\infty\end{array}$ & 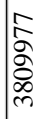 & \begin{tabular}{l}
$\infty$ \\
\multirow{2}{*}{} \\
\multirow{N}{*}{}
\end{tabular} & & & 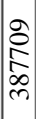 & & 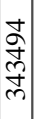 & ڤ્ڤ & $\begin{array}{l}\frac{\pi}{n} \\
\frac{\gamma}{2}\end{array}$ & \\
\hline & $\%$ & $\begin{array}{l}0 \\
\text { ?n. } \\
0\end{array}$ & $\begin{array}{l}\stackrel{0}{\infty} \\
\dot{0}\end{array}$ & ले & ?.? & $\frac{\infty}{0}$ & & $: \mid$ & 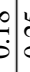 & $\frac{\infty}{c}$ & & 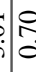 & & & ? & 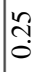 & $\frac{0}{0}$ & $\hat{\sigma}$ & $\begin{array}{l}0 \\
? \\
0 \\
0\end{array}$ & $\begin{array}{l}\stackrel{q}{0} \\
\dot{0}\end{array}$ & $\begin{array}{l}\hat{6} \\
\dot{m}\end{array}$ & $\begin{array}{l}0 \\
⿱ \\
i \\
i\end{array}$ & $\begin{array}{l}\vec{b} \\
\dot{0}\end{array}$ & \pm & \&. & $\stackrel{\overbrace{}}{c}:$ & $\begin{array}{c}y \\
y \\
c\end{array}$ & $\begin{array}{c}n \\
n \\
n\end{array}$ & 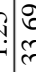 & $\hat{2}$ & $\approx$ & $\frac{n}{6}$ & $\stackrel{2}{\circ}$ & & & & & $\begin{array}{l}+ \\
\dot{0}\end{array}$ & $\bar{?}$ & ". & $\begin{array}{l}2 \\
\vdots \\
i\end{array}$ \\
\hline & $\stackrel{\partial}{\vec{d}} \Xi$ & 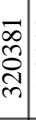 & . & & 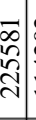 & 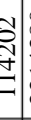 & 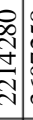 & 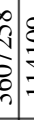 & 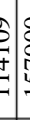 & 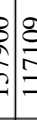 & $=0$ & 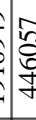 & वृ & & 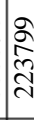 & 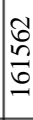 & \begin{tabular}{l}
$\infty$ \\
$\stackrel{0}{0}$ \\
\hdashline \\
\hdashline \\
\hdashline
\end{tabular} & 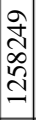 & 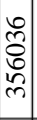 & 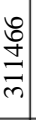 & $\begin{array}{l}\stackrel{o}{+} \\
\vec{\Xi} \\
\vec{\imath}\end{array}$ & 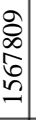 & 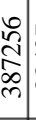 & $\begin{array}{c}0 \\
\tilde{c} \\
\substack{\infty \\
\infty}\end{array}$ & $\begin{array}{l}0 \\
0 \\
0 \\
0 \\
q \\
q\end{array}$ & $\frac{n}{b}$ & 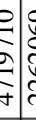 & 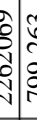 & $\hat{\overbrace{}}$ & ț & $\left\{\begin{array}{l}n \\
\infty \\
\infty \\
\infty\end{array}\right.$ & $\begin{array}{l}8 \\
\frac{8}{5} \\
\text { of } \\
+\end{array}$ & 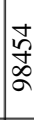 & $\begin{array}{l}\infty \\
\frac{\infty}{\circ} \\
\curvearrowleft \\
n\end{array}$ & & 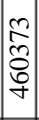 & - & $\begin{array}{l}\infty \\
\infty \\
\hat{0} \\
0 \\
0 \\
+\end{array}$ & 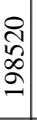 & $\begin{array}{l}\infty \\
\infty \\
n \\
n \\
\infty \\
m\end{array}$ & 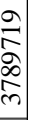 \\
\hline & 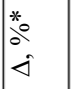 & \begin{tabular}{l}
$\infty$ \\
$\infty$ \\
$\infty$ \\
\hdashline \\
\end{tabular} & 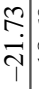 & & 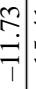 & $\begin{array}{l}\overrightarrow{7} \\
\dot{r} \\
\end{array}$ & 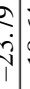 & 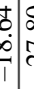 & $\underset{1}{0}$ & is & & $\bar{\top}$ & $\frac{0}{1}$ & ๙̃ & के & 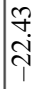 & $\mid \begin{array}{l}f \\
f \\
i \\
i\end{array}$ & 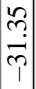 & $\begin{array}{l}\stackrel{g}{0} \\
\vec{i}\end{array}$ & 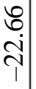 & 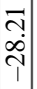 & $\begin{array}{c}\overrightarrow{3} \\
\infty \\
\infty\end{array}$ & & & $=$ & \begin{tabular}{c|c}
0 \\
0 \\
0 \\
\\
1
\end{tabular} & $=$ & 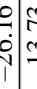 & & & 象 & $\stackrel{\infty}{\stackrel{\infty}{+}}$ & \begin{tabular}{l}
$\infty$ \\
\multirow{N}{*}{} \\
$\stackrel{\sim}{T}$
\end{tabular} & ले & & & & $\frac{\infty}{\tau}$ & 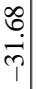 & 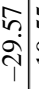 & 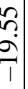 \\
\hline & $*^{*}$ & $\begin{array}{l}\vec{s} \\
\vec{s} \\
\stackrel{1}{1}\end{array}$ & 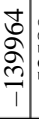 & & & & 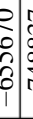 & $\begin{array}{ll}6 \\
0 \\
0 \\
0 \\
0 \\
1\end{array}$ & 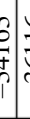 & : & $\left\{\begin{array}{l}0 \\
\vdots \\
\vdots\end{array}\right.$ & $\left\{\begin{array}{l}n \\
\infty \\
0 \\
1\end{array}\right.$ & $\frac{\pi}{\infty}$ & $\frac{\mathfrak{c}}{0}$ & $\stackrel{n}{\infty}$ & $\frac{\vec{\Omega}}{\vec{\gamma}}$ & 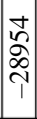 & \begin{tabular}{l}
$m$ \\
$\widehat{\Omega}$ \\
8 \\
\multirow{\gamma}{f}{} \\
\end{tabular} & 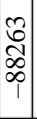 & 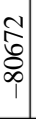 & 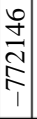 & $\begin{array}{l}\infty \\
\stackrel{0}{2} \\
\stackrel{+}{+} \\
\end{array}$ & 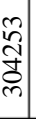 & 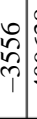 & 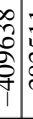 & 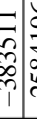 & 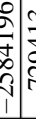 & $\begin{array}{l}n \\
⿱ \\
⿱ \\
1 \\
1\end{array}$ & 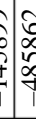 & $\begin{array}{c}0 \\
\infty \\
\infty \\
\infty \\
\infty\end{array}$ & 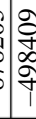 & 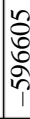 & ڤે & \begin{tabular}{l}
$\infty$ \\
$\infty$ \\
$\infty$ \\
\multirow{1}{1}{} \\
\end{tabular} & \begin{tabular}{l}
$\infty$ \\
\multirow{N}{0}{} \\
$\mathbb{ర}$ \\
\hdashline \\
\end{tabular} & 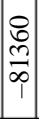 & & \begin{tabular}{l}
0 \\
$\vdots$ \\
$\vdots$ \\
\multicolumn{1}{c}{} \\
\hdashline
\end{tabular} & $\begin{array}{c}\stackrel{t}{n} \\
\stackrel{n}{1}\end{array}$ & 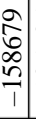 & 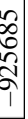 \\
\hline$\stackrel{\mathscr{2}}{=}$ & $\because 0$ & ले & $\stackrel{8}{\circ}$ & तै & है. & \pm & : & $\vec{f}=$ & 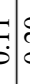 & 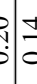 & $P$ & in & 0 & $\mathfrak{c}$ & 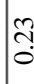 & o. & $\overrightarrow{0}$ & -7 & $\stackrel{+}{\circ}$ & $\stackrel{n}{?}$ & $\mid$ & $\stackrel{\partial}{i}$ & $\mathrm{C}$ & $\frac{m}{0}$ & S & c & $\begin{array}{l}5 \\
0 \\
0 \\
0\end{array}$ & $\begin{array}{c} \pm \\
\mathrm{i}\end{array}$ & 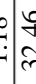 & if & 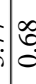 & \& & $=$ & $\stackrel{\infty}{0}$ & & $\mid \begin{array}{l}\infty \\
n \\
0 \\
0\end{array}$ & & סे & ঙ̊. & $\stackrel{\infty}{+}$ & $\begin{array}{l}\infty \\
\dot{v} \\
\dot{v}\end{array}$ \\
\hline $\begin{array}{l}\text { Eี } \\
\text { 娄 }\end{array}$ & 옹 & 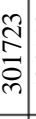 & in & & & 氙 & 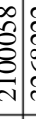 & 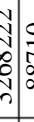 & $\begin{array}{c}2 \\
0 \\
0 \\
0 \\
0\end{array}$ & 2 & a & כે & E & $\frac{n}{n}$ & 。 & $\frac{1}{\Xi}$ & 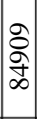 & 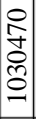 & 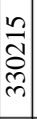 & 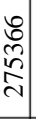 & 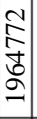 & 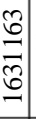 & 尺े & $\begin{array}{l} \\
\triangleright \\
\stackrel{2}{\circ} \\
\curvearrowright\end{array}$ & 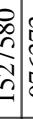 & $\frac{n}{6}$ & 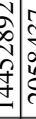 & 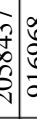 & $\begin{array}{l}0 \\
0 \\
0 \\
0 \\
\\
\end{array}$ & $\begin{array}{l}\hat{b} \\
\hat{n}\end{array}$ & 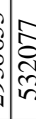 & \begin{tabular}{l}
$\overline{0}$ \\
$\overline{0}$ \\
\multirow{2}{*}{} \\
$\forall$
\end{tabular} & $\begin{array}{l}\hat{\infty} \\
\tilde{N} \\
\infty\end{array}$ & 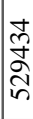 & & 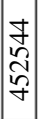 & 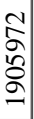 & 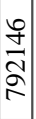 & 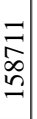 & & . \\
\hline & $\stackrel{*}{\circ}$ & $\underset{Ð}{\ddagger}$ & $\bar{Z}$ & $\bar{m}$ & సิ & $\frac{n}{0}$ & है. & $\begin{array}{l}+ \\
+ \\
\dot{j}\end{array}$ & + & $\overrightarrow{\mathrm{s}}$ & 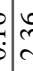 & 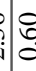 & है & $\frac{2}{2}$ & ? & กิ & $\frac{m}{0}$ & 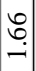 & đ. & సे. & $\begin{array}{l}m \\
\tilde{m}\end{array}$ & $\hat{\alpha}$ & 0 & $=$ & I & in & 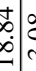 & 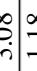 & 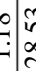 & $?$ & - & $\frac{n}{n}$ & $\frac{0}{0}$ & $\overbrace{0}^{\circ}$ & 10 & 0 & 2 & $=$ & 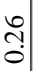 & है: & m? \\
\hline & $\stackrel{a}{\vec{d}}=$ & 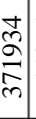 & 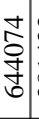 & & & & है & $\frac{\delta}{5}$ & v & $t$ & s & ले & ल & 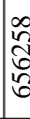 & $\stackrel{\widehat{\infty}}{\infty}$ & & 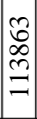 & $\tilde{0}$ & $\begin{array}{l}\frac{\infty}{\tilde{f}} \\
\infty \\
\vec{\sigma} \\
\end{array}$ & 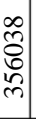 & 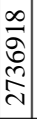 & 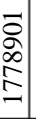 & 守 & जิ & $\frac{\infty}{2}$ & $\begin{array}{l}+ \\
\infty \\
\infty \\
\infty \\
m \\
m\end{array}$ & $\frac{\infty}{2}$ & $\begin{array}{l}0 \\
0 \\
0 \\
0\end{array}$ & $\begin{array}{l}0 \\
0 \\
0 \\
0 \\
0\end{array}$ & th & 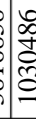 & $\begin{array}{l}\frac{0}{\pi} \\
\frac{n}{n} \\
\frac{n}{n}\end{array}$ & 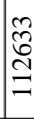 & $\begin{array}{l}\text { I } \\
\text { d } \\
\end{array}$ & & & ح̊ & 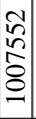 & 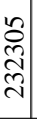 & & \\
\hline 栉 & & 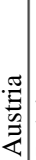 & 言 & & 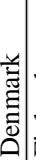 & $\pi$ & & & & & & & & & 票 & & 部 & & & & 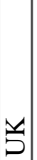 & & & & & 8 & & & & & & & 芠 & స్ & : & \begin{tabular}{|l|}
$\vec{\alpha}$ \\
$\vdots$ \\
$\bar{z}$
\end{tabular} & $\mid \begin{array}{c}0 \\
0 \\
0 \\
\vdots \\
0 \\
0 \\
0\end{array}$ & 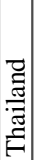 & 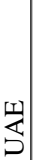 & 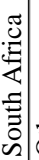 & \\
\hline
\end{tabular}


Table 3. Classification of the countries of the world by the destructive effects of the pandemic crisis of 2020 for the automotive industry

\begin{tabular}{|c|c|c|c|c|c|c|c|c|c|c|}
\hline 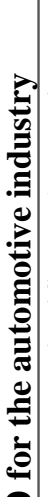 & 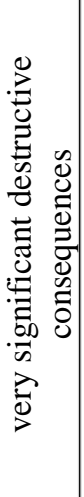 & & 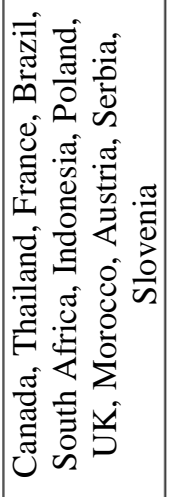 & 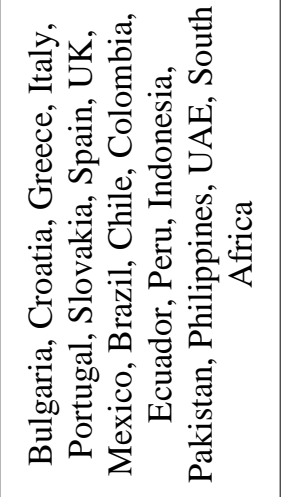 & & 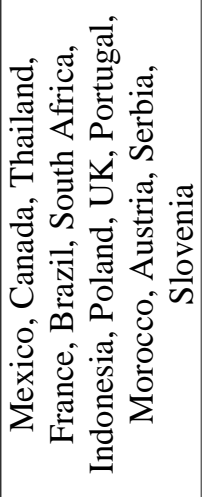 & 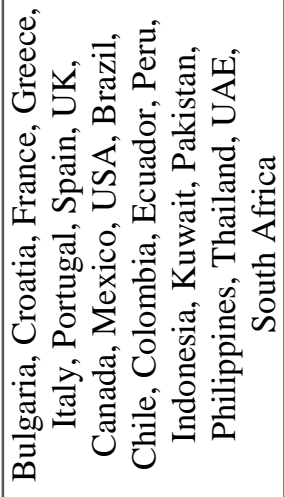 & & 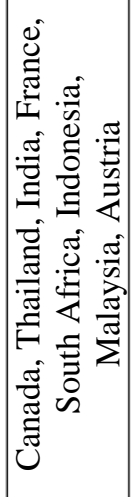 & 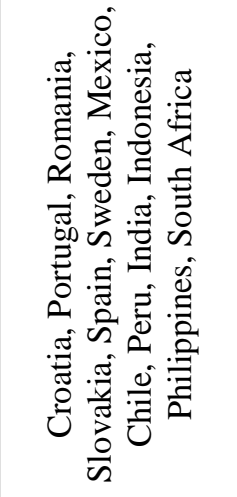 \\
\hline 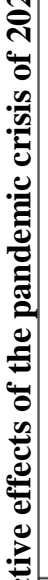 & 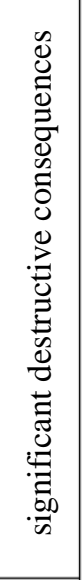 & $\mid \begin{array}{l}\tilde{0} \\
\frac{0}{0} \\
0 \\
0 \\
0 \\
0\end{array}$ & 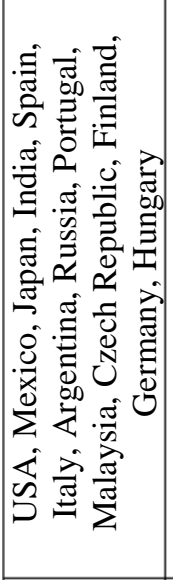 & 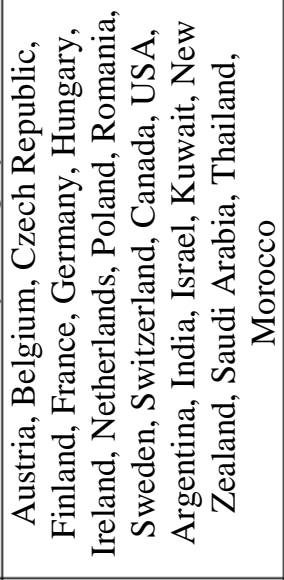 & 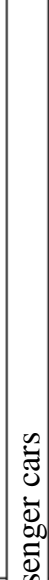 & 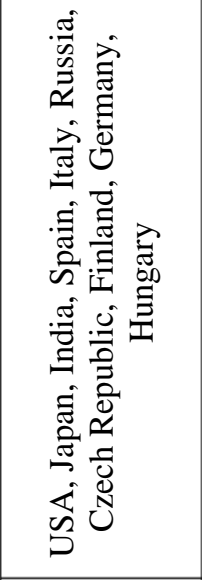 & 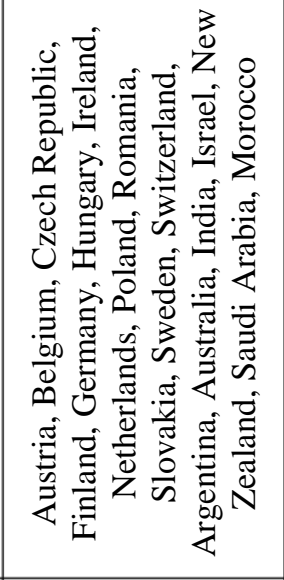 & $\begin{array}{l}\frac{0}{0} \\
: \frac{0}{0} \\
0 \\
\frac{7}{0} \\
\cdot \frac{\pi}{0}\end{array}$ & 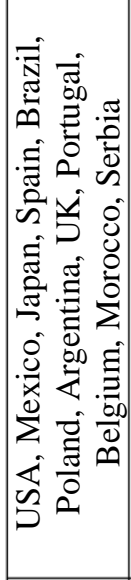 & 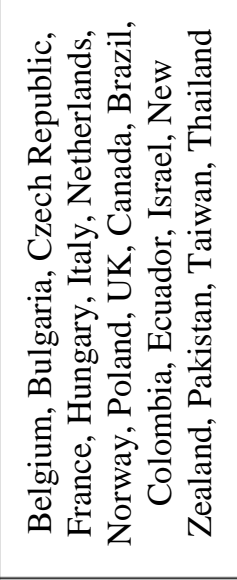 \\
\hline 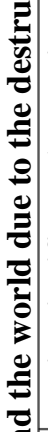 & 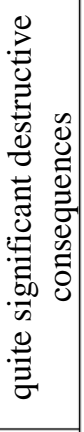 & 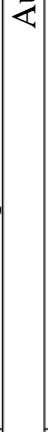 & 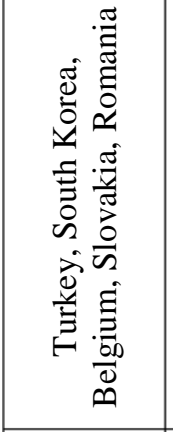 & 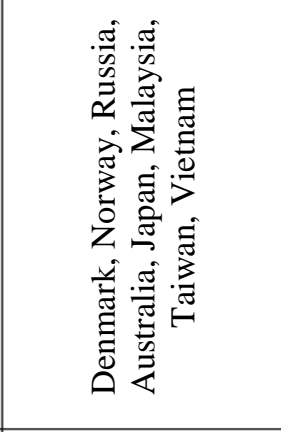 & డ & 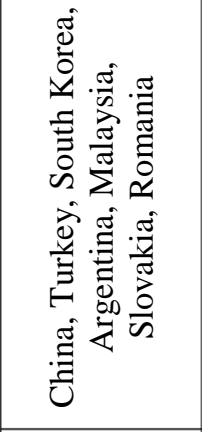 & 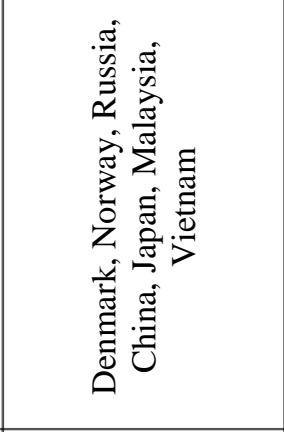 & $\begin{array}{l}\text { Z्g } \\
\text { 己े }\end{array}$ & 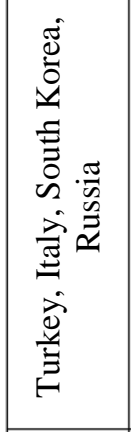 & 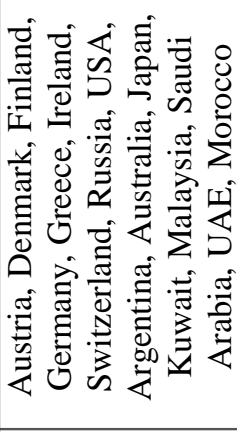 \\
\hline 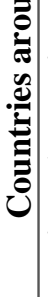 & 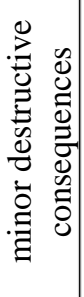 & & 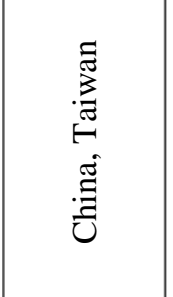 & 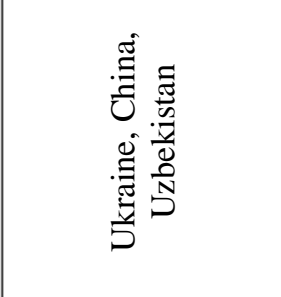 & & 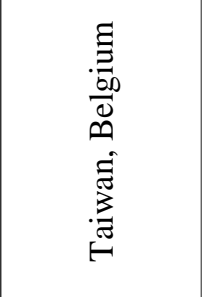 & 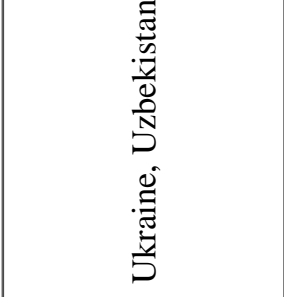 & & 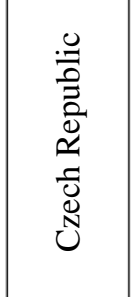 & 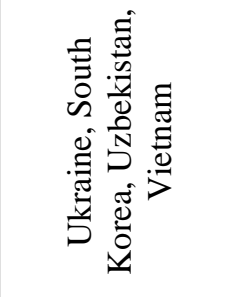 \\
\hline & 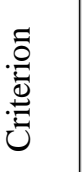 & & $\begin{array}{l}\frac{0}{0} \\
0 \\
\frac{0}{0} \\
0 \\
0\end{array}$ & $\frac{\mathscr{U}}{\tilde{\pi}}$ & & 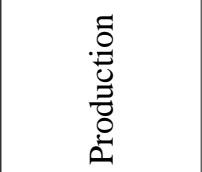 & $\underset{\mathscr{N}}{\tilde{N}}$ & & 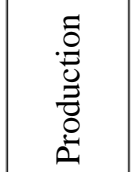 & $\stackrel{\pi}{\pi}$ \\
\hline
\end{tabular}


In 2020, there were some growing trends in car sales, in particular, in Turkey (by $61.85 \%$, including cars - by $57.55 \%$, commercial vehicles - by $77.75 \%$ ), Kazakhstan (by $30.02 \%$, including cars - by $30.1 \%$, commercial vehicles by $23.04 \%$ ), Egypt (by $28.82 \%$, including cars by $31,66 \%$, commercial vehicles - by $20.44 \%$ ), South Korea (by $6.17 \%$, including cars - by $8.1 \%$ ), Puerto Rico (by $1.53 \%$, including cars by $1.73 \%$, commercial vehicles - by $0.68 \%$ ). Also in Taiwan, sales of passenger cars increased by $19.97 \%$, and in China - sales of commercial vehicles increased by $18.69 \%$.

\section{Conclusion}

Based on the conducted vertical and horizontal analysis of car production and sales in the world, the impact of the COVID-19 pandemic on the automotive industry has been determined. The results of the vertical analysis of car production in the world give us the opportunity to state that the flagships of the global automotive industry are China, USA, Japan, Germany, India, Mexico, South Korea, Brazil, Spain, and France. Based on the results of the horizontal analysis of car production and sales in the world, we will classify the countries of the world by the destructive effects of the pandemic crisis of 2020 for the automotive industry by criterion of reducing car production and sales (Table 3), where the reduction: by a percentage not exceeding $5 \%$, can be identified as minor destructive effects; by a percentage in the range of 5-15\% - as quite significant destructive consequences; by a percentage in the range of 15-25\% - as significant destructive consequences; by a percentage exceeding $25 \%-$ as very significant destructive consequences.

The obtained results can be recommended in identifying further ways to overcome the negative effects of the COVID-19 pandemic in the automotive industry.

\section{Conflict of interests}

The authors state that there is no conflict of interest regarding the publication of this article.

\section{References}

1. Долбнєва Д. В. (2020). Вплив COVID-19 на економіку країн світу. Проблеми економіки, 1 (43), 20-26. Dolbnieva, D. V. (2020). Vplyv COVID-19 na ekonomiku krain svitu [COVID-19 injection into the economy of the country]. Economy problems, 1 (43), 20-26. [in Ukrainian]. https://doi.org/10.32983/2222-0712-2020-1-2026
2. Зара А. С. (2020). Розвиток автомобілебудівної індустрії в умовах пандемії: основні чинники та перспективи. Науковий вісник Ужгородського національного університету. Серія: Міжнародні економічні відносини та світове господарство, 32, 12-19. Zara, А. Ye. (2020). Rozvytok avtomobilebudivnoi industrii v umovakh pandemii: osnovni chynnyky ta perspektyvy. [Development of the automotive industry in a pandemic: the main factors and prospects]. Scientific Bulletin of Uzhhorod National University. Series: International Economic Relations and the World Economy, 32, 12-19. [in Ukrainian]

3. Вплив COVID-19 та карантинних обмежень на економіку України (2020). Центр прикладних досліджень. Vplyv COVID-19 ta karantynnykh obmezhen na ekonomiku Ukrainy (2020) [Impact of COVID-19 and quarantine restrictions on the economy of Ukraine] Center for Applied Research.

4. Report: The impact of road transport sector regulation on the entrepreneurship and economic growth in the European Union. Motor Transport Institute. Warsaw-LinzLyon, February 2020, available at: https://www.mobilelabour.eu/wpcontent/uploads/2018/02/The-Impact-of-

Regulation-of-the-Road-Transport-Sector-onEntreperneurship-and-Economic-Growth.pdf (last accessed 21.09.2021).

5. Adlah, A., Taghreed, M., Zaabi, E., Haton E. (2021). Impact of COVID-19 on Entrepreneurship and Consumer Behaviour: A Case Study in Saudi Arabia. The Journal of Asian Finance, Economics and Business, 8 (5), 201-210.

https://doi.org/10.13106/jafeb.2021.vol8.no5.020

6. Puriwat, W., Tripopsakul, S. (2021). Customer Engagement with Digital Social Responsibility in Social Media: A Case Study of COVID-19 Situation in Thailand. Journal of Asian Finance, Economics and Business, 8 (2), 475-483. https://doi.org/10.13106/jafeb.2021.vol8.no2.0475

7. Giones, F., Brem, A., Pollack, J. M., Michaelis, T. L., Klyver, K., Brinckmann, J. (2020). Revising entrepreneurial action in response to exogenous shocks: Considering the COVID-19 pandemic. Journal of Business Venturing Insights, 14. e00186.

https://doi.org/10.1016/j.jbvi.2020.e00186

8. Impacts of the COVID-19 pandemic on EU industries, available at: https://www.europarl.europa.eu/RegData/etudes/ STUD/2021/662903/IPOL_STU(2021)662903_E N.pdf (last accessed 21.10.2021)

9. Iveta Kufelova, I., Rakova, M. (2020). Impact of the Covid-19 pandemic on the automotive industry in Slovakia and selected countries. S SHS Web Conf. Volume 83, Current Problems of the Corporate Sector 2020. https://doi.org/10.1051/shsconf/20208301040

10. Janmenjoy, N., Manohar, M., Bighnaraj, N., Hanumanthu, S., Korhan, C. \& Vimal, Sh. (2021). 
An impact study of COVID-19 on six different industries: Automobile, energy and power, agriculture, education, travel and tourism and consumer electronics. Wiley Public Health Emergency Collection, PMC8014102. doi: 10.1111/exsy.12677

11. Nicola, M., Alsafi, Z., Sohrabi, C., Kerwan, A., alJabir, A., Iosifidis, C., Agha, M., \& Agha, R. (2020). The socio-economic implications of the coronavirus pandemic (COVID-19): A review. International Journal of Surgery (London, England), 78, 185-193.

12. Rajamohan, S., Sathish, A., \& Rahman, A. (2020). Impact of COVID-19 on stock price of NSE in automobile sector. The International Journal of Advanced Multidisciplinary Research, 7(7), 2429.

13. Yan, Y., et al. (2020). An empirical study on consumer automobile purchase intentions influenced by COVID-19. Available at SSRN 3593963.

14. Production Statistics. International Organization of Motor Vehicle Manufacturers, available at: https://www.oica.net/production-statistics/

15. Sales Statistics. International Organization of Motor Vehicle Manufacturers. available at: https://www.oica.net/category/sales-statistics/

Inna Shevchenko ${ }^{1}$, DSci (Economics), Assoc. Prof., Dean of Faculty of Management and Business, e-mail: shevchenko.khnadu@gmail.com, tel.: +38-066-187-28-30,

ORCID: 0000-0003-0758-9244

Illia Dmytriiev ${ }^{1}$, DSci (Economics), Prof.

Vice-rector for scientific work,

dmitriev.khnadu@gmail.com,

tel.: +38-050-587-97-66,

ORCID: 0000-0001-8693-3706

Oksana Dmytriieva1, DSci (Economics), Assoc.

Prof., Head of the Department of Economics and

Business, oksanahnadu@gmail.com,

tel. +38-063-353-79-98,

ORCID: 0000-0001-9314-350X

${ }^{1}$ Kharkiv National Automobile and Highway

University Yaroslava Mudrogo str., 25, Kharkiv,

Ukraine, 61002

\section{Вплив пандемії COVID-19 на виробництво та} продажі автомобілів у світі

Анотація. Проблема. Світова автомобільна індустрія вже має досвід відновлення після глобальної фінансової кризи 2008 року, проте пандемічна криза 2020 року є зовсім іншою за природою та характером перебігу: в новітній історії вона ще не мала аналогів $і$ поки що передчасною буде констатація ї̈ завершення. Тому важливим питанням $є$ визначення впливу пандемї на виробництво та продаж автомобілів для подолання негативних наслідків. Для вирімення цьього питання у статті визначено чутливість иієї підгалузі машинобудування до деструктивних змін у зовнішньому середовищ;; проведено аналіз зміни обсягів виробництва та продажу автомобілів за краӥнами світу за останній період. Мета. Метою роботи $\epsilon$ визначення деструктивних наслідків та тендениій виливу пандемії COVID-19 на світову автомобільну індустрію, а саме виробництво та продаж автомобілів. Методологія. В роботі для визначення впливу пандемії COVID-19 проведено вертикальний $i$ горизонтальний аналіз виробництва та продажі автомобілів у світі. Результати. Отримані результати аналізу дозволили авторам провести групування країн світу за силою деструктивних наслідків пандемічної кризи 2020 року для автомобільної індустрії. Орихінальність. Проведене групування країн за силою деструктивних наслідків пандемії COVID-19 надали можливість отримати уявлення про його вплив на автомобільну галузь, зокрема виробництво та продаж автомобілів. Практичне значення. Отримані результати можуть бути рекомендовані при для визначенні подальших шляхів подолання негативних наслідків пандемї COVID-19 у автомобільній індустрії.

Ключові слова: автомобільна індустрія, соціально-економічний розвиток, виробництво автомобілів, продаж автомобілів, пандемія COVID-19.

Шевченко Інна Юріївна ${ }^{1}$, д.е.н., доцент, декан факультету управління та бізнесу, shevchenko.khnadu@gmail.com, тел.: +38-066-187-28-30, ORCID: 0000-0003-0758-9244

Дмитрієв Ілля Андрійович ${ }^{1}$, д.е.н., професор, проректор з наукової роботи, заслужений діяч науки і техніки України,

dmitriev.khnadu@gmail.com, тел.: +38-050-587-97-66,

ORCID: 0000-0001-8693-3706

Дмитрісва Оксана Іллівна ${ }^{1}$, д.е.н., доцент, завідувач кафедри економіки і підприємництва, oksanahnadu@gmail.com, тел. +38-063-353-79-98, ORCID: 0000-0001-9314-350X

${ }^{1}$ Харківський національний автомобільнодорожній університет, вул. Ярослава Мудрого, 25, м. Харків, Україна, 61002. 